\title{
Molecularly Imprinted Cyclodextrin Polymers as Stationary Phases of High Performance Liquid Chromatography
}

\author{
Takayuki HishiYa, ${ }^{* \dagger}$ Hiroyuki Asanuma, ${ }^{*, *,+\dagger}$ and Makoto KomIYAMA ${ }^{*}, \dagger$ \\ *Research Center for Advanced Science and Technology, \\ The University of Tokyo, 4-6-1 Komaba, Meguro-ku, Tokyo 153-8904, Japan \\ ** Precursory Research for Embryonic Science and Technology (PRESTO), \\ Japan Science and Technology Corporation (JST), Kawaguchi 332-0012, Japan
}

(Received January 14, 2003; Accepted March 10, 2003)

\begin{abstract}
Imprinted cyclodextrin polymers are prepared and applied to stationary phases of high performance liquid chromatography (HPLC) for the selective recognition of steroids and other hydrophobic guest molecules. Molecularimprinting efficiently promotes the binding-affinity and substrate-selectivity towards the template molecules compared with the control polymers prepared in their absence. When rigid and hydrophobic molecules such as cholesterol are imprinted, retention times towards the template molecules are notably enhanced. Thus, imprinting of cyclodextrins is effective for the preparation of synthetic tailor-made receptors for various kinds of hydrophobic guest molecules, and the polymers are potentially applicable to selective recognition of biologically important molecules or removal of toxic molecules from aqueous media.
\end{abstract}

KEY WORDS Cyclodextrin / Molecular Imprinting / Steroids / High Performance Liquid Chromatography (HPLC) / Stationary Phase /

Regulation of molecular assembly and its orientation has been the central interest in supramolecular chemistry ${ }^{1}$ and molecular recognition. ${ }^{2}$ Organization of hosts such as calixarene, ${ }^{3}$ cyclophane, ${ }^{4}$ and cyclodextrins $^{5}$ (CyDs) is a key technique for the selective recognition of nano-sized guest molecules (e.g., steroids, sugars, peptides, nucleic acids). Since these guest molecules are too large for a single host compound to efficiently accommodate, cooperation of plural hosts is required to employ efficient binding activity. Breslow et al. reported that cyclodextrin dimer ${ }^{6}$ and trimer efficiently recognize steroids, ${ }^{7}$ peptides, ${ }^{8}$ and other biologically important molecules. ${ }^{9}$ Cooperative binding by two CyD molecules linked with a proper linker is essential for efficient binding (and catalytic) activities.

Although these host assemblies are very effective for molecular recognition, they require elaborate synthetic procedure. Thus, a simple and versatile method to organize host assemblies in a tailor-made fashion is very attractive and important in the field of molecular recognition. One of the promising methodologies for their purpose is molecular imprinting. ${ }^{10}$ This technique is based on 1) adduct formation between functional monomer(s) and a template molecule, 2) immobilization by radical polymerization, and 3 ) removal of template molecules from the polymer. Complementary structure to the target formed in the polymer functions as guest-binding site. Molecular imprinting of conven- tional vinyl monomers is widely investigated to prepare chemical sensors, separation carrier, artificial antibody catalyst, and so on. ${ }^{11,12}$

We have recently applied this technique to the preparation of CyD assembly as schematically illustrated in Figure 1 for the selective recognition of steroids in aqueous media. ${ }^{13-15}$ It was found that imprinted CyD polymers prepared in the presence of cholesterol bind the template cholesterol more efficiently and selectively than the random CyD assembly prepared in its absence. The structure of binding sites was also clarified by physicochemical analysis. ${ }^{15}$

In the present study, molecularly imprinted polymers are applied to high performance liquid chromatography (HPLC) stationary phases by using steroids and other highly hydrophobic guests as templates. The imprinted CyD polymers efficiently and selectively retain the template molecules. Application of this method to the selective separation of steroids or dioxin models from aqueous media is also discussed.

\section{EXPERIMENTAL}

\section{Materials and Methods}

Cyclodextrins (CyDs), steroids, hydrophobic guests, and cross-linking agents were purchased from Tokyo Kasei Co. Ltd. and used without further purification. Dimethyl sulfoxide (DMSO) was dried with molecu-

\footnotetext{
${ }^{\dagger}$ Present Address: Institute of Materials Chemistry and Engineering, Kyushu University, Fukuoka 812-8581, Japan.

${ }^{\dagger \dagger}$ To whom correspondence should be addressed (E-mail: asanuma@mkomi.rcast.u-tokyo.ac.jp, komiyama@mkomi.rcast.u-tokyo.ac.jp).
} 


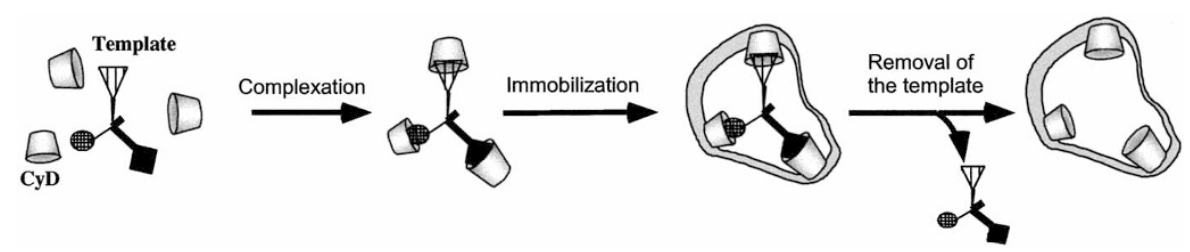

Figure 1. Schematic illustration of molecular imprinting of cyclodextrins (CyDs) for the preparation of tailor-made receptors.

lar sieve 4A and then distilled under a reduced pressure. Steroids and CyDs were dried in vacuo at $70^{\circ} \mathrm{C}$ for $24 \mathrm{~h}$ before use. Water was purified by a Millipore Milli-XQ purification system. A stainless steel column $(4.6 \mathrm{~mm} \times 5.0 \mathrm{~cm})$ and packer $(50 \mathrm{~mL})$ were purchased from GL Science Co. Ltd.

\section{Preparation of Imprinted CyD Polymers and Packing into Stainless Steel Columns as Liquid Chromatogra- phy Stationary Phases}

$\beta$-CyD was cross-linked with toluene diisocyanate as reported before. ${ }^{15 \mathrm{a}}$ In dry DMSO, $\beta$-CyD $(88 \mathrm{mM})$ was cross-linked with toluene 2,4-diisocyanate (TDI) (560 $\mathrm{mM}$ unless otherwise noted) in the presence of a template $(44 \mathrm{mM})$. After being magnetically stirred at $65^{\circ} \mathrm{C}$ for $2 \mathrm{~h}$, the gel formed was chopped into pieces, washed with acetone, and ground with mortar and pestle. The polymer was sufficiently washed with acetone, tetrahydrofuran (THF), and hot water to remove the template molecule, CyDs, and cross-linker. Then, the polymer was dried in vacuo at $70^{\circ} \mathrm{C}$ for $24 \mathrm{~h}$. Thorough removal of the template molecules was confirmed by ${ }^{13} \mathrm{C} \mathrm{CP} / \mathrm{MAS}$ NMR. Non-imprinted $\mathrm{CyD}$ polymers (control polymers) were also prepared in the same manner as described above except for the absence of template molecules. The polymer obtained was further finely ground with mortar and pestle, and then particles with the size between $38-63 \mu \mathrm{m}$ were collected by using stainless sieves. The powder was added to an aqueous acetonitrile (AN) solution (AN/water = $50 / 50(\mathrm{v} / \mathrm{v}))$ and suspended by an ultrasonic sonicator for $10 \mathrm{~min}$. After being incubated for $10 \mathrm{~min}$, the supernatant of the suspension was removed. This sonicationsuspension procedure was repeated three times, and then the residue was packed into a stainless steel column by using a stainless packer $(50 \mathrm{~mL})$. The control (non-imprinted) polymer, which was prepared in the absence of the template, was also similarly treated and packed into a column for the measurement.

\section{Measurement of Retention Times of Substrates}

The column obtained was first eluted with acetonitrile until the base line became stable. A mixture of water and acetonitrile was used as eluent to measure retention times by UV detector $(\lambda=220 \mathrm{~nm}$ and $260 \mathrm{~nm}$ for steroids and aromatic compounds, respectively) ${ }^{16}$
Table I. Retention times of cholesterol-imprinted and non-imprinted $\beta$-CyD polymers cross-linked with TDI

\begin{tabular}{lllc}
\hline \multirow{2}{*}{ Guests } & \multicolumn{2}{l}{ Retention time $\left(\mathrm{t}-\mathrm{t}_{0}\right) / \mathrm{min}^{\mathrm{a}}$} & \multirow{2}{*}{ Imp/Non } \\
\cline { 2 - 3 } & $\operatorname{Imp}$ & Non & \\
\hline Cholesterol & 3.13 & 0.13 & 24 \\
Stigmasterol & 0.91 & 0.56 & 1.6 \\
Prognenolone & 3.01 & 0.65 & 5.4 \\
Progesterone & 1.83 & 0.90 & 2.0 \\
4-Cholesten-3-one & 0.80 & 0.27 & 3.0 \\
\hline
\end{tabular}

${ }^{\mathrm{a}} \mathrm{t}_{0}$ : Retention time of acetone (void marker). Eluent : wa$\operatorname{ter} / \mathrm{AN}=5 / 95(\mathrm{v} / \mathrm{v})$.

Flow rate was $0.5 \mathrm{~mL} \mathrm{~min}^{-1}$ and acetone was used as a void marker. The retention times, measured twice, were identical with each other within 0.1 min. $^{17}$

\section{RESULTS AND DISCUSSION}

\section{Promotion of the Retention Time toward Cholesterol by its Imprinting}

Table I shows retention behaviors of the cholesterolimprinted and non-imprinted $\beta$-CyD polymers as stationary phases by use of water/acetonitrile $(\mathrm{AN})=$ $5 / 95(\mathrm{v} / \mathrm{v})$ as eluent. When cholesterol was used as a template, the retention time for the template (cholesterol) was efficiently promoted by the imprinting compared with that of non-imprinted polymer (from 0.13 to $3.1 \mathrm{~min}$, see Table I). ${ }^{17}$ Promotion of the retention time (Imp/Non) was as high as 24 . This high imprinting effect toward cholesterol is attributed to the formation of dimeric CyDs as a binding site during the polymerization..$^{5 \mathrm{~b}, 18}$ When other steroids were used on cholesterol-imprinted polymer, these values were much smaller (below 6): retention times toward 4cholesten-3-one and stigmasterol were not much promoted, though they have similar structure to cholesterol. These chromatographic results are consistent with those obtained from batch-wise adsorption assay reported before. ${ }^{15}$ Relatively high Imp/Non ratio for pregnenolone $(\mathrm{Imp} / \mathrm{Non}=5.4)$ is attributed to the similarity of the steroid framework between the template cholesterol and pregnenolone. When stigmasterol was used as a template in place of cholesterol, the retention time for stigmasterol was promoted (from $0.56 \mathrm{~min}$ to $3.3 \mathrm{~min}$ ) under the same HPLC conditions employed. Thus, it can be concluded that imprinting of $\beta$-CyD is 
Table II. Effect of water ratio in eluent on the promotion of binding by cholesterol-imprinting ${ }^{\text {a }}$

\begin{tabular}{|c|c|c|c|c|}
\hline \multirow{2}{*}{ Guests } & \multicolumn{4}{|c|}{ Imp/Non ratio } \\
\hline & \multicolumn{2}{|c|}{$0 / 100$} & $5 / 95$ & $10 / 90$ \\
\hline Cholesterol & 0.19 & $(0.54)^{\mathrm{c}}$ & 24 & 27 \\
\hline Stigmasterol & 0.21 & $(0.42)$ & 1.6 & 5.3 \\
\hline Pregnenolone & 1.3 & $(0.84)$ & 5.4 & 11 \\
\hline Progesterone & 1.6 & $(2.74)$ & 2.0 & 3.9 \\
\hline 4-Cholesten-3-one & 2.3 & $(0.79)$ & 3.0 & 7.4 \\
\hline
\end{tabular}

${ }^{a}$ Ratio of the retention time $\left(\mathrm{t}-\mathrm{t}_{0}\right)$ of the guest by the cholesterol-imprinted polymer with respect to that by the non-imprinted control polymer. ${ }^{\text {b Ratio }}$ of water and acetonitrile (v/v) in eluent. ${ }^{c}$ Values in parentheses are retention times of the non-imprinted $\mathrm{CyD}$ polymer.

effective for the preparation of the polymer selective for the template. When $\alpha$-CyD was used as a host molecule instead of $\beta$-CyD, the column obtained did not retain the steroids at all (data not shown). This result clearly shows that guest molecules were bound to $\beta$-CyD assemblies formed by the imprinting, not to the cavity formed by the cross-linking molecules.

\section{Effects of Eluents on Retention Behavior}

In order to assess the contribution of hydrophobic interaction to the binding, effect of eluent on retention behaviors was studied by varying the water content in the eluent (Table II). When the eluent contained some water, Imp/Non ratio was eminent only for cholesterol (see columns of 5/95 and 10/90 (v/v) in Table II). In case of water/AN $=0 / 100(\mathrm{v} / \mathrm{v})$ where hydrophobic interaction does not work at all, however, imprinting effect was completely diminished. Rather, hydrogen-bonding dominated the retention behavior. The binding activities toward guest molecules depend on the number of hydrogen-bonding sites in each molecule (see Figure 2 and the parentheses in Table II). Pregnenolone (2.7 min), which has two carbonyl groups, was bound most strongly to $\beta$-CyD in the non-imprinted polymer by hydrogen-bonding with its hydroxyl groups. The second strongest was progesterone (2.0 min), which has one carbonyl and one hydroxyl group. 4-Cholesten-3one $(0.79 \mathrm{~min})$ was weakly bound because it has one carbonyl group. Cholesterol $(0.84 \mathrm{~min})$ and stigmasterol $(0.42 \mathrm{~min})$ were bound with least affinity since they have only one hydroxyl group and no carbonyl groups. In water $/ \mathrm{AN}=5 / 95$ or $10 / 90(\mathrm{v} / \mathrm{v})$, where hydrogen bonding is destroyed by the competition with water and hydrophobic interaction is dominant, selectivity of the imprinted polymer toward cholesterol became efficient. Thus, imprinting effect of cholesterol can be attributed to inclusion complex formation, not to hydrogen bonding. As the ratio of AN in eluent decreased (from water/AN $=5 / 95$ to $10 / 90(\mathrm{v} / \mathrm{v})$ ), Imp/Non ratio for steroids other than cholesterol much
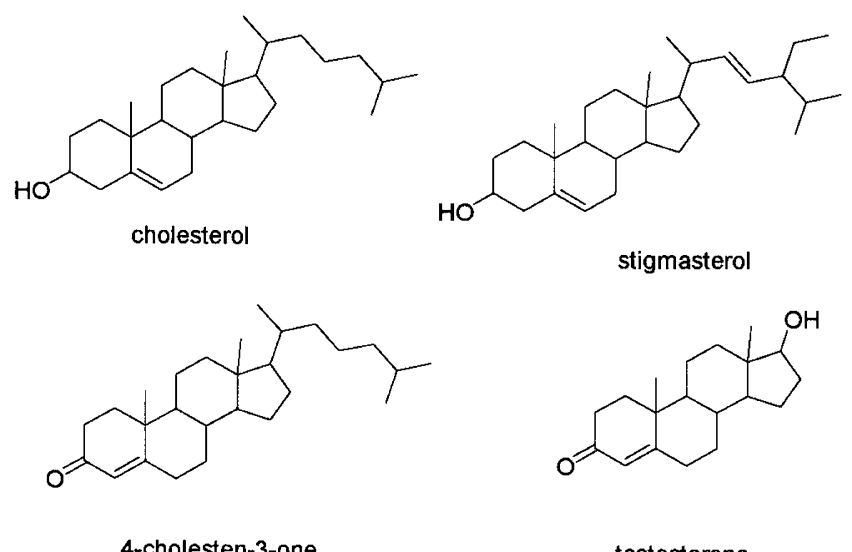

4-cholesten-3-one

testosterone
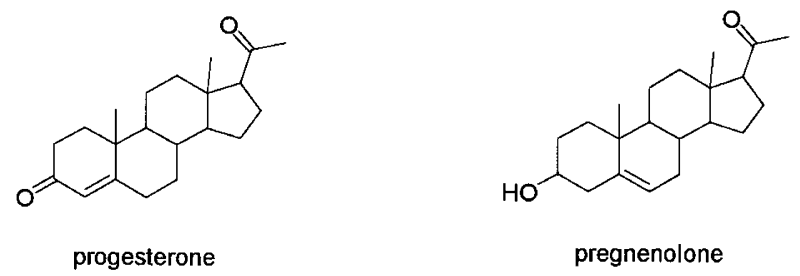

Figure 2. Chemical structures of steroids used as templates and guests in the present study.

increased, resulting in the decrease in the selectivity. This is because at a high content of AN, only strong binding sites dominate the retention behavior, while weak binding sites as well as strong ones participate in the recognition at a lower content.

\section{Effect of the Concentration of Cross-Linker on the Im- printing}

As described in the previous section, eminent imprinting was observed when $[\mathrm{TDI}] /[\mathrm{CyD}]$ ratio was $6.4([\mathrm{TDI}]=560 \mathrm{mM})$. However, increase in the TDI concentration $([\mathrm{TDI}]=1120 \mathrm{mM})$ significantly interfered the imprinting. As shown in Table III, Imp/Non was rather small (compare Table I with Table III) and substrate-selectivity of the polymers toward cholesterol against stigmasterol was nil. High cross-linkage ratio may restrict the flexibility essential for the selective binding. In fact, selectivity of the polymers was 
Table III. Retention times $\left(\mathrm{t}-\mathrm{t}_{0}\right)$ of cholesterol-imprinted and non-imprinted $\beta$-CyD polymer prepared with high concentration of $\mathrm{TDI}^{\mathrm{a}, \mathrm{b}}$

\begin{tabular}{|c|c|c|c|c|c|c|}
\hline \multirow{3}{*}{ Guests } & \multicolumn{3}{|c|}{ water/AN $=20 / 80$} & \multicolumn{3}{|c|}{ water/AN $=40 / 60$} \\
\hline & \multicolumn{2}{|c|}{$\overline{\text { Retention time / min }}$} & \multirow{2}{*}{ Imp/Non } & \multicolumn{2}{|c|}{ Retention time / min } & \multirow{2}{*}{ Imp/Non } \\
\hline & $\operatorname{Imp}$ & Non & & $\operatorname{Imp}$ & Non & \\
\hline Cholesterol & 2.1 & 0.76 & 2.8 & 15.0 & 5.8 & 2.6 \\
\hline Stigmasterol & 2.3 & 0.84 & 2.7 & 18.0 & 6.9 & 2.6 \\
\hline Pregnenolone & 2.1 & 1.0 & 2.1 & 3.9 & 3.0 & 1.3 \\
\hline Progesterone & 2.1 & 1.7 & 1.2 & 5.9 & 5.0 & 1.3 \\
\hline 4-Cholesten-3-one & 2.1 & 1.7 & 1.2 & 20.1 & 9.4 & 2.1 \\
\hline
\end{tabular}

${ }^{\mathrm{a}}[\mathrm{TDI}]=1120 \mathrm{mM}([\mathrm{TDI}] /[\beta-\mathrm{CyD}]=12.7) .{ }^{\mathrm{b}}$ Retention time was very short in eluent with high content $(>90 \%)$ of $\mathrm{AN}$.

Table IV. Template effect on the promotion of retention behavior of HPLC stationary phases

\begin{tabular}{|c|c|c|}
\hline Template & Imp/Non & Template effect \\
\hline Cholesterol $^{\mathrm{a}}$ & 27 & very high \\
\hline Progesterone $^{\mathrm{a}}$ & 1.1 & nil \\
\hline Phenoxathiin $^{\mathrm{b}}$ & 2.0 & high \\
\hline D1(amide compound) $)^{a, c}$ & 1.0 & nil \\
\hline $\mathrm{D} 2^{\mathrm{a}, \mathrm{c}}$ & 1.0 & nil \\
\hline
\end{tabular}

${ }^{\mathrm{a}}$ Eluent : water/AN $=10 / 90(\mathrm{v} / \mathrm{v}) .{ }^{\mathrm{b}}$ Eluent $:$ water $/ \mathrm{AN}=$ 25/75 (v/v). ${ }^{\mathrm{c}}$ See Figure 3 for the structure.

higher when a flexible cross-linker (hexamethylene diisocyanate) was used. ${ }^{15}$

\section{Requisite of Templates for the Effective Imprinting}

Table IV shows the retention behaviors of the imprinted polymers prepared with various hydrophobic templates. Not all the hydrophobic template molecules were effective for the imprinting: templates having rigid molecular structure (such as steroids and phenoxathiin, etc.) were effectively imprinted (high Imp/Non ratio), whereas flexible compounds in which two aromatic rings are connected with polymethylene chain (e.g., amide compounds in Figure 3) did not exhibit imprinting effect. This fact demonstrates that sufficient rigidity is required for the template to immobilize the organized CyDs strictly in the polymer. When templates were rigid enough, hydrophobic molecules of similar structure could be discriminated by the imprinting, even if the templates did not have functional groups. As shown in Table V, the polymer imprinted with $p$-terphenyl retained the template ( $p$-terphenyl) most strongly among three hydrophobic molecules tested here $(3.68 \mathrm{~min})$. As expected, $\mathrm{m}$ terphenyl- and trans-stilbene-imprinted CyD polymers selectively and preferentially bound them, respectively. Complex formation of CyDs with these rigid templates contributes to the imprinting effect. ${ }^{19}$

\section{Application to the Recognition of Dioxin Model}

Today, highly hydrophobic compounds such as dioxin arouse one of the most crucial environmen-
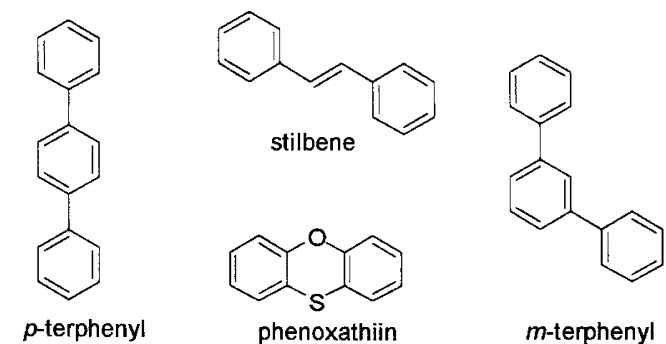

$m$-terphenyl

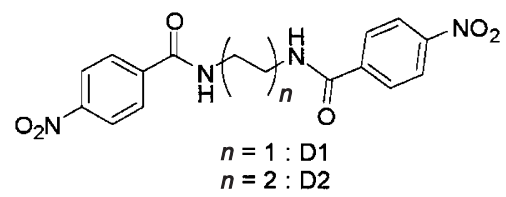

Figure 3. Chemical structures of highly hydrophobic compounds used in this study.

Table V. Retention behavior of hydrophobic rigid guests

\begin{tabular}{lccc}
\hline $\begin{array}{l}\text { Polymer } \\
\text { (template })\end{array}$ & \multicolumn{3}{c}{ Retention time $\left(\mathrm{t}-\mathrm{t}_{\mathrm{o}}\right) / \mathrm{min}^{\mathrm{a}}$} \\
\cline { 2 - 4 } trans-stilbene & $m$-terphenyl & $p$-terphenyl \\
\hline $\mathrm{P}($ trans -stilbene $)$ & 3.55 & 1.83 & 1.22 \\
$\mathrm{P}(m$-terphenyl $)$ & 1.54 & 3.03 & 1.04 \\
$\mathrm{P}(p$-terphenyl $)$ & 1.87 & 1.57 & 3.68 \\
\hline${ }^{\mathrm{a}} \mathrm{t}_{\mathrm{o}}:$ Retention & time of acetone. & Eluent : & water $/ \mathrm{AN}=$ \\
$25 / 75(\mathrm{v} / \mathrm{v})$. & & &
\end{tabular}

tal problems. However, selective removal of these molecules is difficult because they have no functional groups available for precise molecular recognition. Moreover, these molecules have no hydrogen-bonding site so that it is difficult to apply conventional imprinting technique. Hydrophobic interaction, which is usually non-selective, can be used for the selective recognition of guests by using the present molecular imprinting. Here, we applied this method to the recognition of hydrophobic molecules other than steroids. ${ }^{20}$ A dioxin model (phenoxathiin: Figure 3) was used as template molecules as harmful compounds to be removed. ${ }^{21}$ When phenoxathiin was used as a template, the retention time for the template molecule was greatly enhanced by the imprinting (from 3.68 to $7.31 \mathrm{~min}$, see Table VI), whereas retention times of other molecules 
Table VI. Retention behaviors of phenoxathiin-imprinted and non-imprinted $\beta$-CyD polymer ${ }^{\text {a }}$

\begin{tabular}{lcc}
\hline \multirow{2}{*}{ Guest } & \multicolumn{2}{c}{ Retention time (t) / min } \\
\cline { 2 - 3 } & Phenoxathiin-Imp & Non \\
\hline m-Terphenyl & 1.45 & 1.32 \\
trans-Stilbene & 1.66 & 1.52 \\
Phenylalanine & 1.73 & 1.55 \\
4-Phenylazoaniline & 1.79 & 1.60 \\
4-Nitrophenol & 1.88 & 1.61 \\
Phenylazonaphthalene & 1.57 & 1.65 \\
Phthalimide & 2.04 & 1.68 \\
p-Terphenyl & 1.83 & 1.76 \\
Methyl benzoate & 1.62 & 1.81 \\
Azobenzene & 1.87 & 1.81 \\
4-Nitroaniline & 2.07 & 1.99 \\
Cumene & 2.16 & 2.06 \\
Biphenyl & 2.42 & 2.06 \\
2,2-Dipyridyl & 2.66 & 2.33 \\
Dibenzofuran & 3.35 & 2.41 \\
Phenoxathiin & 7.31 & 3.68 \\
\hline
\end{tabular}

${ }^{\mathrm{a}}$ Measured in water $/ \mathrm{AN}=10 / 90(\mathrm{v} / \mathrm{v})$.

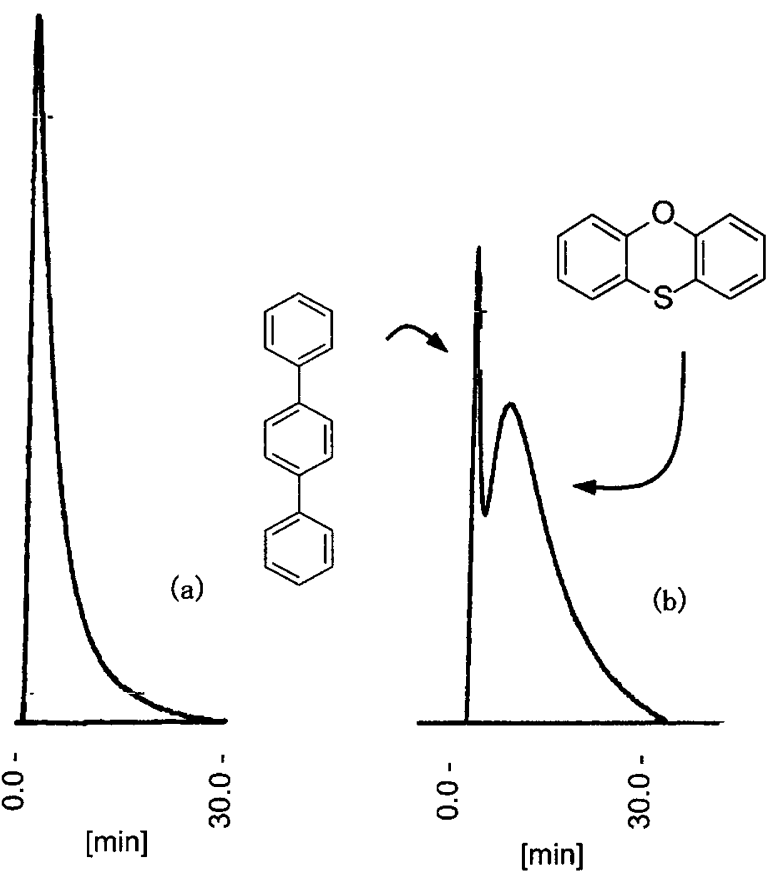

Figure 4. Retention behaviors toward a mixture of phenoxathiin and $p$-terphenyl retained by (a) non-imprinted and (b) phenoxathiin-imprinted $\mathrm{CyD}$ polymers at $25^{\circ} \mathrm{C}$. Eluent is a mixture of water/AN $=50 / 60(\mathrm{v} / \mathrm{v})$.

were essentially unchanged. Thus, selective promotion of binding by the imprinting was successful. For the non-imprinted $\mathrm{CyD}$ polymers, the retention times of the phenoxathiin and $p$-terphenyl were almost the same, so that the mixtures of phenoxathiin and $p$ terphenyl could not be separated (Figure 4a). On the other hand, imprinted column could selectively bind phenoxathiin (Figure 4b). These results demonstrate that present imprinting is applicable to the templates without hydrogen-bonding interaction.

\section{CONCLUSIONS}

Imprinting of $\beta$-CyD is effective in regulating $\mathrm{CyD}$ molecules to cooperatively bind the target molecules. Application of this method to the preparation of HPLC stationary phases was successful using steroids and other hydrophobic guests as templates. Hydrophobic interaction, which is usually non-selective, can be used as a selective and cooperative interaction by the present imprinting. This method has a potentiality to prepare tailor-made stationary phases for the separation of highly hydrophobic compounds such as dioxin derivatives. Besides CyDs, modified CyDs and other host molecules may be promising as recognition sites for still more efficient and selective artificial receptors in a tailor-made fashion.

Acknowledgment. We thank Professor Toshifumi Takeuchi (Kobe Univ.) and Dr. Jun Matsui (Konan Univ.) for valuable advice on column chromatography. This work was partially supported by Grants-inAid for Scientific Research from the Ministry of Education, Science, Sports and Culture, Japan. The supports by JSPS Research Fellowships for Young Scientists (for T. H.), Tokuyama Science Foundation (for H. A.), and The Ogasawara Foundation for the Promotion of Science and Engineering (for H. A.) are also acknowledged.

\section{REFERENCES AND NOTES}

1. J.-M. Lehn, "Supramolecular Chemistry", VCH, Weinheim, 1995.

2. M. M. Conn and J. Rebek, Jr., Chem. Rev., 97, 1647 (1997).

3. a) J. Vicens and V. Bohmer, Ed., "Calixarenes: A Versatile Class of Macrocyclic Compounds", Kluwer Academic Publishers, Dordrecht, 1991.

b) J. Vicens, Z. Asfari, and J.M. Harrowfield, Ed., "Calixarenes 50th Anniversary: Commemorative Volume", Kluwer Academic Publishers, Dordrecht, 1994.

c) H. S. Park, Q. Lin, and A. D. Hamilton, J. Am. Chem. Soc., 121, 8 (1999).

4. P. Wallimann, T. Marti, A. Furer, and F. Diederich, Chem. Rev., 97, 1567 (1997).

5. a) M. L. Bender and M. Komiyama, "Cyclodextrin Chemistry”, Springer-Verlag GmbH \& Co, Berlin 1978.

b) J. Szèjtlí, “Cyclodextrin Technology”, Kluwer Academic Publishers, Budapest (1988).

c) K. A. Connors, Chem. Rev., 97, 1325 (1997).

d) A. Harada, Adv. Polym. Sci., 133, 141 (1997).

6. M. R. de Jong, J. F. J. Engbersen, J. Huskens, and D. N. Reinhoudt, Chem. Eur. J., 6, 4034 (2000).

7. a) Z. Yang and R. Breslow, Tetrahedron Lett., 38, 6171 (1997). 
b) R. Breslow and B. Zhang, J. Am. Chem. Soc., 118, 8495 (1996).

8. R. Breslow, Z. W. Yang, R. Ching, G. Trojandt, and F. Odobel, J. Am. Chem. Soc., 120, 3536 (1998).

9. D. K. Leung, Z. W. Yang, and R. Breslow, Proc. Natl. Acad. Sci. U.S.A., 97, 5050 (2000).

10. a) K. Haupt and K. Mosbach, Trends Biotechnol., 16, 468 (1998).

b) K. Mosbach, Trends Biochem. Sci., 19, 9 (1994).

11. a) D. A. Spivak and K. J. Shea, Macromolecules, 31, 2160 (1998).

b) K. J. Shea and D. Y. Sasaki, J. Am. Chem. Soc., 113, 4109 (1991).

c) M. J. Whitcombe, M. E. Rodriguez, P. Villar, and E. N. Vulfson, J. Am. Chem. Soc., 117, 7105 (1995).

d) B. Sellergren, J. Wieschemeyer, K. S. Boos, and D. Seidel, Chem. Mater, 10, 4037 (1998).

e) B. Sellergren, Angew. Chem., Int. Ed. Eng., 39, 1031 (2000).

f) M. E. Davis, A. Katz, and W. R. Ahmad, Chem. Mater, , 8, 1820 (1996).

g) O. Ramström and K. Mosbach, Curr. Opin. Chem. Biol., 3, 759 (1999).

h) G. Wülff, Angew. Chem., Int. Ed. Eng., 34, 1812 (1995).

i) T. Takeuchi and J. Haginaka, J. Chromatogr. B, 728, 1 (1999).

j) J. Matsui, Y. Miyoshi, O. Doblhoff-Dier, and T. Takeuchi, Anal. Chem., 67, 4404 (1995).

12. a) C. J. Allender, K. R. Brain, and C. M. Heard, "Progress in Medicinal Chemistry", 2nd ed, F. D. King and A. W. Oxford, Ed., Elsevier Science Publishers B. V., Amsterdam, 1999. vol. 36, chapt. 6, p 235.

b) J. U. Klein, M. J. Whitcombe, F. Mulholland, and E. N. Vulfson, Angew. Chem., Int. Ed. Eng., 38, 2057 (1999).

c) V. T. Remcho and Z. J. Tan, Anal. Chem., 71, 248 A (1999).

13. a) H. Asanuma, T. Hishiya, and M. Komiyama, Adv. Mater., 12, 1019 (2000).

b) H. Asanuma, T. Akiyama, K. Kajiya, T. Hishiya, and M. Komiyama, Anal. Chim. Acta, 435, 25 (2001).

c) H. Asanuma, K. Kajiya, T. Hishiya, and M. Komiyama, Chem. Lett., 665 (1999).

14. S. A. Piletsky, H. S. Andersson, and I. A. Nicholls, Macromolecules, 32, 633 (1999).

15. a) T. Hishiya, M. Shibata, M. Kakazu, H. Asanuma, and M. Komiyama, Macromolecules, 32, 2265 (1999).

b) T. Hishiya, H. Asanuma, and M. Komiyama, J. Am. Chem. Soc., 124, 570 (2002).

16. At a higher ratio of water in the eluent, peaks were too broadened to determine retention times precisely.

17. Present imprinting made the peak broadened as observed in the conventional imprinting. But, it did not affect the measurement of retention times for the guests at all.

18. High hydrophobicity of cholesterol compared with other steroids also contributed to this remarkable imprinting effect.

19. NOE was observed between $\mathrm{CyD}$ molecules and the guest ( $p$-terphenyl) in DMSO, indicating that complex was formed during polymerization. UV spectrum was also changed (peak shift was observed) on addition of $\beta$-CyD in DMSO.

20. This method has an advantage in preparing artificial receptors for the chemicals sparingly soluble in water.

21. Phenoxathiin is similar in structure to dioxin compounds except for the lack of halogen atoms. 